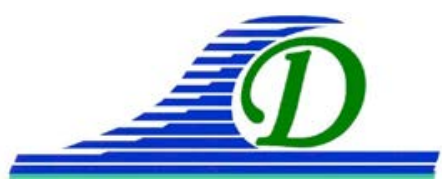

XIII İmes Journées Nationales Génie Côtier - Génie Civil

Dunkerque, 2-4 juillet 2014

DOI:10.5150/jngcgc.2014.048 (c) Editions Paralia CFL

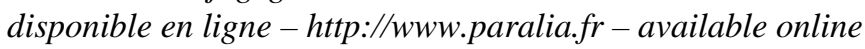

\title{
Expérimentation de chasses hydrauliques à Saint-Valery-sur-Somme
}

\author{
Sophie LEPRETRE ${ }^{1}$
}

1. Conseil Général de la Somme, Direction de la Modernisation des Infrastructures, Service Pilotage de Projets, 85, rue Roger Dumoulin - 80026 Amiens cedex 1, France.s.lepretre@somme.fr

\section{Résumé :}

En cours d'expérimentation, le projet de chasses hydrauliques à Saint-Valery-surSomme vise, en faisant monter de manière maîtrisée le niveau d'eau dans un Canal maritime de $17 \mathrm{~km}$ de linéaire, à créer un effet de chasse permettant d'arracher, puis d'emmener les sédiments au large de manière à favoriser les effets d'autocurage dans le chenal de navigation des ports de Saint-Valery-sur-Somme.

Mots clés : Chasses hydrauliques, Effets de chasse, Auto curage, Protocole de chasses, Dépôt sédimentaire, Impact environnemental, Diagnostic morphologique, Analyse écologique, Espèces faunistiques, Faune benthique, Suivi de l'estran, Cyprinidés, Etat sédimentologique et hydrobiologique, Suivi piscicole.

Key-words: Hydraulic flushing, Effects of hydraulic flushing, Self cleaning, Flushing protocol, Sedimentary deposits, Environmental impact, Morphological diagnosis, Ecological analysis, Wildlife species, Benthic fauna, Foreshore monitoring, Cyprinids, Semimentological and hydrobiological state, Fish monitoring.

\section{Introduction}

Le Département de la Somme est propriétaire et gestionnaire du canal de la Somme et de la Somme canalisée ainsi que des trois ports de pêche en baie de Somme. Les premières réflexions du projet global "Baie de Somme", qui ont commencé dès 1990 (SOGREAH Ingénierie \& EDF, 1994-1997), prévoyaient d'améliorer l'accessibilité à ces trois ports par effet de chasse hydraulique et d'auto curage des chenaux. Pour le site de Saint-Valery, il intégrait aussi l'évacuation des crues de la Somme.

Autorisés par arrêté préfectoral le 14 janvier 2005, des travaux importants d'aménagement et de modernisation ont été réalisés sur les deux barrages de SaintValery-sur-Somme. Ces travaux, financés par le Fond Européen de Développement Régional (FEDER), l'Etat, la Région, l'Agence de l'Eau et le Département ont permis de mettre en place tous les équipements nécessaires pour réaliser des chasses hydrauliques. Pour l'expérimentation des chasses hydrauliques, les financeurs sont l'Etat, la Région et le Département. Dès 2006, le Département de la Somme a confié au bureau d'études ARTELIA la réalisation d'une étude de définition d'un protocole des actions de chasse selon des conditions de débit de la Somme et de marées. 


\section{Thème 2 - Dynamique sédimentaire}

La nécessité d'une phase d'expérimentation avant l'intégration du protocole dans la gestion habituelle des ouvrages a été mise en lumière. Une période d'expérimentation permet d'affiner le protocole initial tout en mesurant l'effet des chasses et d'évaluer également les impacts environnementaux en baie de Somme et dans les biefs amont entre Saint-Valery-sur-Somme et Pont-Rémy (figure 1).

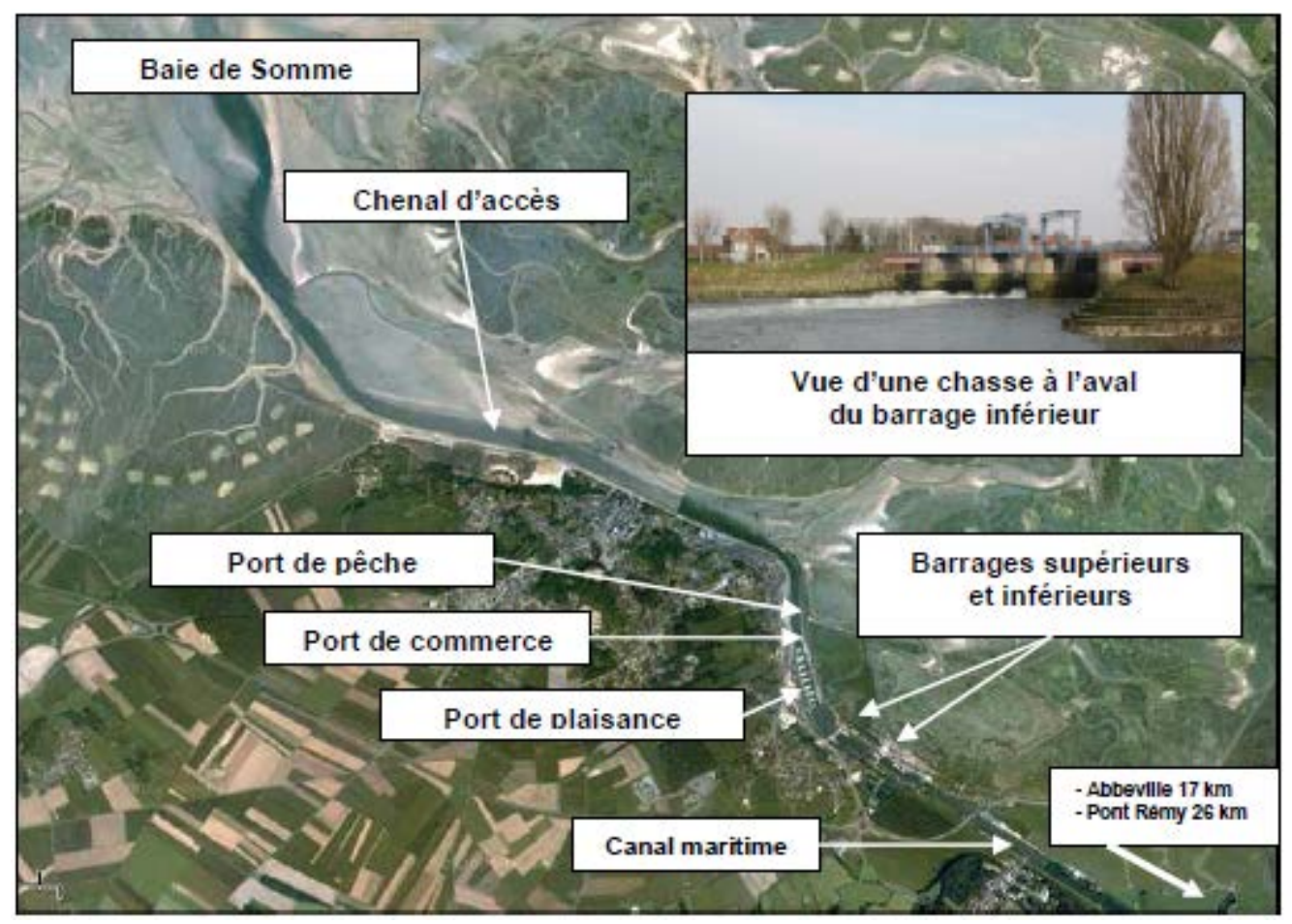

Figure 1. Vue aérienne autour de Saint-Valery-sur-Somme.( SOGREAH Ing., 2007).

2. Mise en place d'un protocole hydraulique et d'un protocole de suivi associé (SOGREAH Ingénierie, 2007).

Une chasse hydraulique est une action volontaire qui consiste à repousser vers le large, à "chasser" des dépôts sédimentaires gênant les écoulements et la navigation. Elle utilise les capacités naturelles des écoulements et ne mobilise aucun engin particulier. C’est une technique naturelle et peu onéreuse de lutte contre l'ensablement. Elle ne peut avoir lieu que dans certaines conditions particulières (marées, débits, météorologie, ...).

Dans cette étude elle vise à remplacer les actions lourdes, coûteuses et administrativement pesantes de dragage d'entretien dans le chenal d'accès et les ports de Saint-Valery-sur-Somme. L'efficacité du procédé vient de la répétition des chasses.

Une gestion spécifique des ouvrages de Saint- Valery-sur-Somme permet de stocker de l'eau dans le Canal maritime entre Saint-Valery- sur-Somme et Abbeville :

- lorsque les conditions de marée le permettent, à marée montante, le barrage inférieur est ouvert afin de permettre le remplissage par l'eau de la baie ; 


\section{XIII ${ }^{\text {èmes }}$ Journées Nationales Génie Côtier - Génie Civil \\ Dunkerque, 2-4 juillet 2014}

- dès que le niveau de sécurité est atteint, le barrage se ferme afin de compléter le stockage du canal par le débit amont ;

- lorsque le niveau d'eau dans le canal est atteint (4,60 m IGN), le barrage passe en régulation jusqu'à ce que la cote de $1,70 \mathrm{~m}$ IGN dans le port soit atteinte, l'ouverture contrôlée du barrage (débit $=110 \mathrm{~m}^{3} / \mathrm{s}$ ) est alors réalisée et un écoulement naturel et puissant se forme et chasse les sédiments dans les ports et le chenal ;

- en fin de chasse, l'ouvrage retrouve son mode normal de fonctionnement.

Cette gestion spécifique prend en compte la gestion de la navigation ainsi que le passage des poissons migrateurs.

Un suivi sur la bathymétrie, l'environnement et les niveaux d'eau est effectué pendant l'expérimentation puis à long terme. Ce suivi a pour objectif de vérifier et analyser les effets des chasses sur différents paramètres et de proposer à la fin des chasses expérimentales un protocole de suivi à long terme.

Le suivi bathymétrique consiste en un levé multifaisceaux de la zone comprise entre le barrage inférieur et le cap Hornu afin de suivre l'évolution des fonds et ainsi de définir la fréquence des chasses adaptée pour le maintien des fonds.

Le suivi environnemental sur la zone d'étude qui s'étend depuis Saint-Valery-surSomme jusqu'à Pont-Rémy est assuré par un groupement de bureau d'études ARTELIA/IN VIVO/FISH PASS et étudie 5 paramètres principaux :

i) L'état des berges, composé :

(a) d'un diagnostic morphologique afin de localiser les dégradations des berges, identifier les causes et les conséquences (observations en rives gauche et droite jusqu'à Pont Rémy),

(b) d'une analyse écologique qui recense les espèces faunistiques et floristiques actuelles caractéristiques dans le but de cerner leurs évolutions du fait des chasses (observations jusqu'à Abbeville en incluant les zones humides adjacentes).

ii) Le suivi de l'estran et des surfaces exondées, qui comprend une analyse des désordres et dépôts vaseux par un diagnostic visuel et la prise de photos aériennes à marée basse (figure 2).

iii) L'état sédimentologique, granulométrique, chimique et hydrobiologique (faune benthique) des sédiments.

iv) L'analyse des vitesses, des mesures de salinité et de la turbidité des masses d'eau.

v) Un suivi piscicole sur le comportement des cyprinidés et l'entraînement de ces espèces ainsi que des civelles à l'amont et l'aval des ouvrages (voir figure 3).

En complément des données récupérées par les sondes de mesures de niveaux automatiques au droit des ouvrages, un suivi des niveaux d'eau est effectué grâce à des relevés sur les échelles limnimétriques au niveau des zones urbanisées à l'amont et plus particulièrement au niveau des exutoires et des secteurs sensibles ayant déjà subi des inondations et grâce à un nivellement de la hauteur d'eau par rapport au haut de berge sur les zones de débordement potentielles identifiées le long du canal. 
Thème 2 - Dynamique sédimentaire

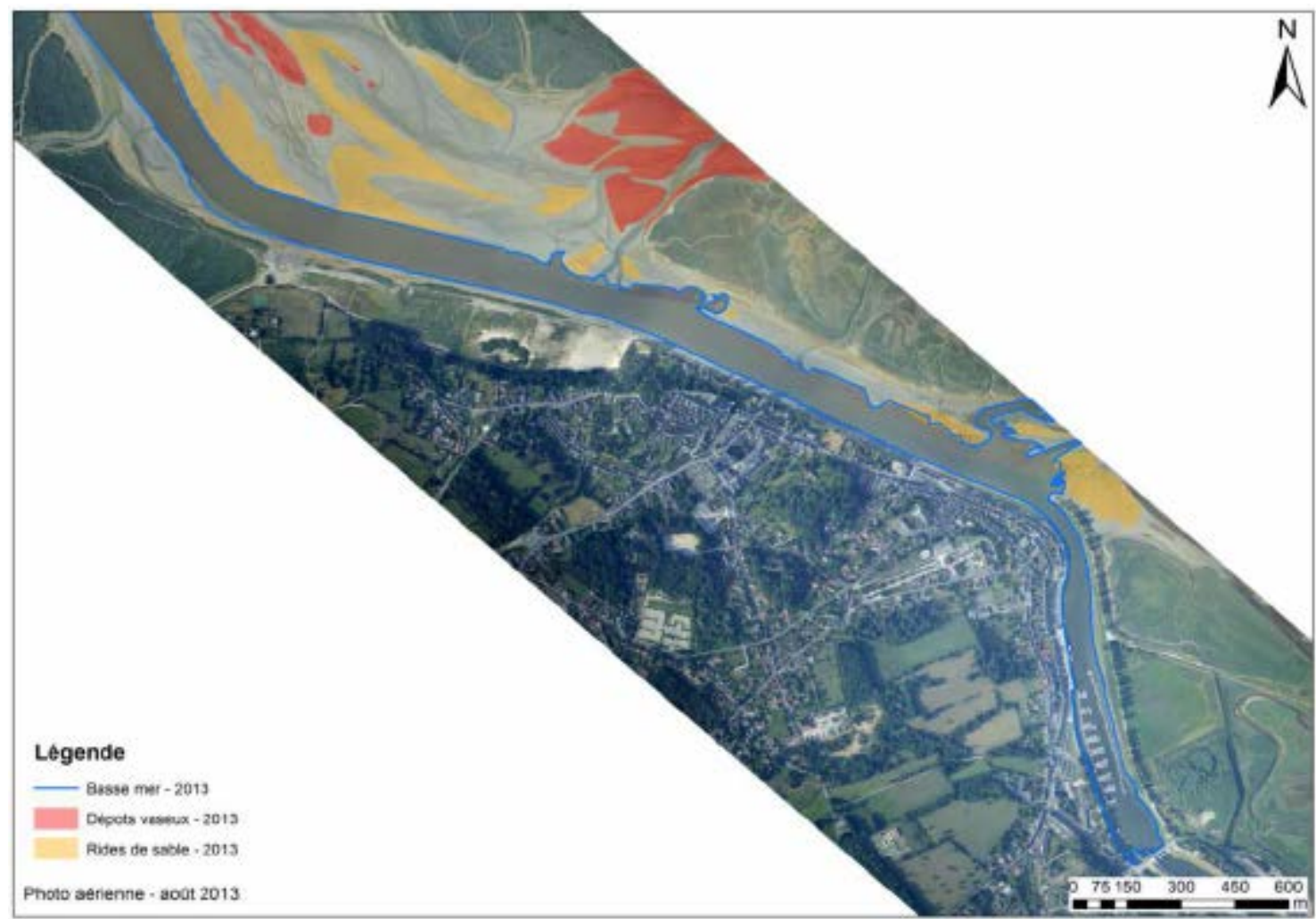

Figure 2. Suivi de l'estran (IN VIVO et al., 2013).

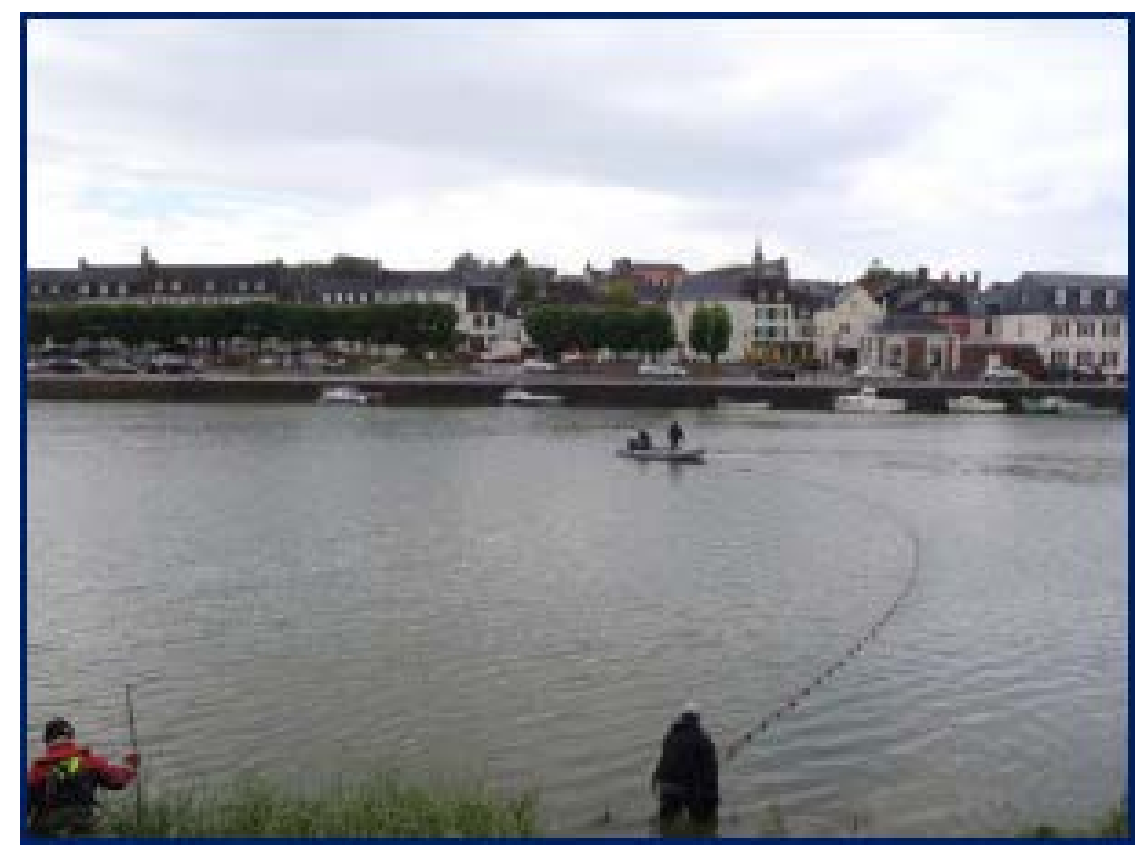

Figure 3. Echantillonnage de pêche (technique de la senne) (IN VIVO et al., 2013). 


\section{XIII ${ }^{\text {èmes }}$ Journées Nationales Génie Côtier - Génie Civil \\ Dunkerque, 2-4 juillet 2014}

\section{Etude expérimentale}

L'expérimentation comporte 4 phases :

i) la phase préliminaire (de mai à septembre 2012) a établi un état de référence sur la zone influencée par les chasses (de Pont-Rémy à la baie de Somme) sur les aspects sédimentologiques et environnementaux ;

ii) la phase 1 "Tests de stockage et de mise en vitesse" (en novembre 2012) consistait en des essais d'élévation progressive du niveau dans le Canal maritime (figure 4) et aux essais de mise en vitesse progressive à l'aval du barrage inférieur :

- les essais de stockage augmentent progressivement la cote de stockage actuelle de 3,50 m IGN jusqu'à la cote admissible de 4,60 m IGN ; ils ont été menés avec une gestion du niveau amont à 4,00 m, 4,30 m, 4,60 m (IGN); un premier essai de remplissage partiel par l'eau de la baie n'a pu être effectué du fait des conditions météorologiques inadéquates ;

- les essais de mise en vitesse (en simultané avec les essais de stockage) visent à augmenter progressivement les courants, compris actuellement entre $0,3 \mathrm{~m} / \mathrm{s}$ et $0,4 \mathrm{~m} / \mathrm{s}$ jusqu'à $1 \mathrm{~m} / \mathrm{s}$ pour la remise en suspension des sédiments à l'aval ; ils ont été menés avec des vitesses de $0,60 \mathrm{~m} / \mathrm{s}, 0,80 \mathrm{~m} / \mathrm{s}$ et $1,00 \mathrm{~m} / \mathrm{s}$;

cette phase a permis de confirmer les résultats établis au cours de l'étude hydraulique initiale, à savoir retenir le niveau 4,60 m IGN comme niveau haut de stockage sans provoquer de désordres sur l'amont et à atteindre des vitesses entre $0,6 \mathrm{~m} / \mathrm{s}$ et $0,8 \mathrm{~m} / \mathrm{s}$ pour permettre "l'arrachage" et le transport des sédiments; les levés bathymétriques ont montré un abaissement généralisé d'environ $20 \mathrm{~cm}$ des fonds du canal, du sas et du port sauf dans quelques secteurs particuliers, ce qui a permis de conclure à l'efficacité des actions de chasses dans le port de SaintValery-sur-Somme ; le suivi des niveaux d'eau et des zones sensibles n'ont pas fait apparaître de débordement, aucun impact notoire sur les milieux n’a par ailleurs été constaté ;

iii) la phase 2 "séquences courtes" (en mars/avril 2013) a comporté des séquences de 4 chasses successives (marées de jour et de nuit) sur 4 marées consécutives ;

ces séquences ont montré que les fonds sont toujours en évolution et que l'état d'équilibre n'est pas atteint sauf peut-être dans le canal et le sas ; la réduction des volumes d'érosion au cours des essais laisse penser que l'état futur des fonds n'est pas nécessairement très loin de l'état atteint (en moyenne); localement (à l'aval des vannes du barrage), les fonds sont fortement sollicités et il est très difficile de déterminer un état futur; il est probable que cette zone évolue en permanence (l'érosion est plus liée à la turbulence locale qu'aux vitesses ellesmêmes); lors de ces essais, il a été noté qu'une fréquence de 4 chasses par semaine conduit à un niveau intéressant d'érosion des fonds; la poursuite des suivis environnementaux n’a montré aucune incidence sur les milieux ; 


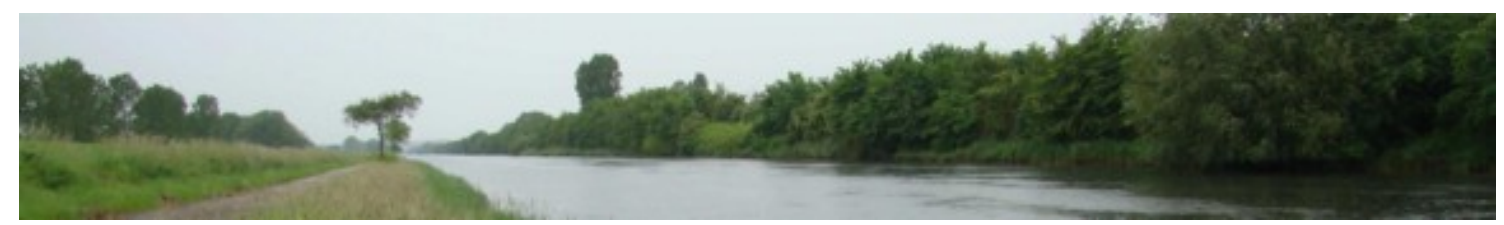

Figure 4. Vue sur Canal maritime - Niveau à 4,60 m IGN. (IN VIVO et al., 2013).

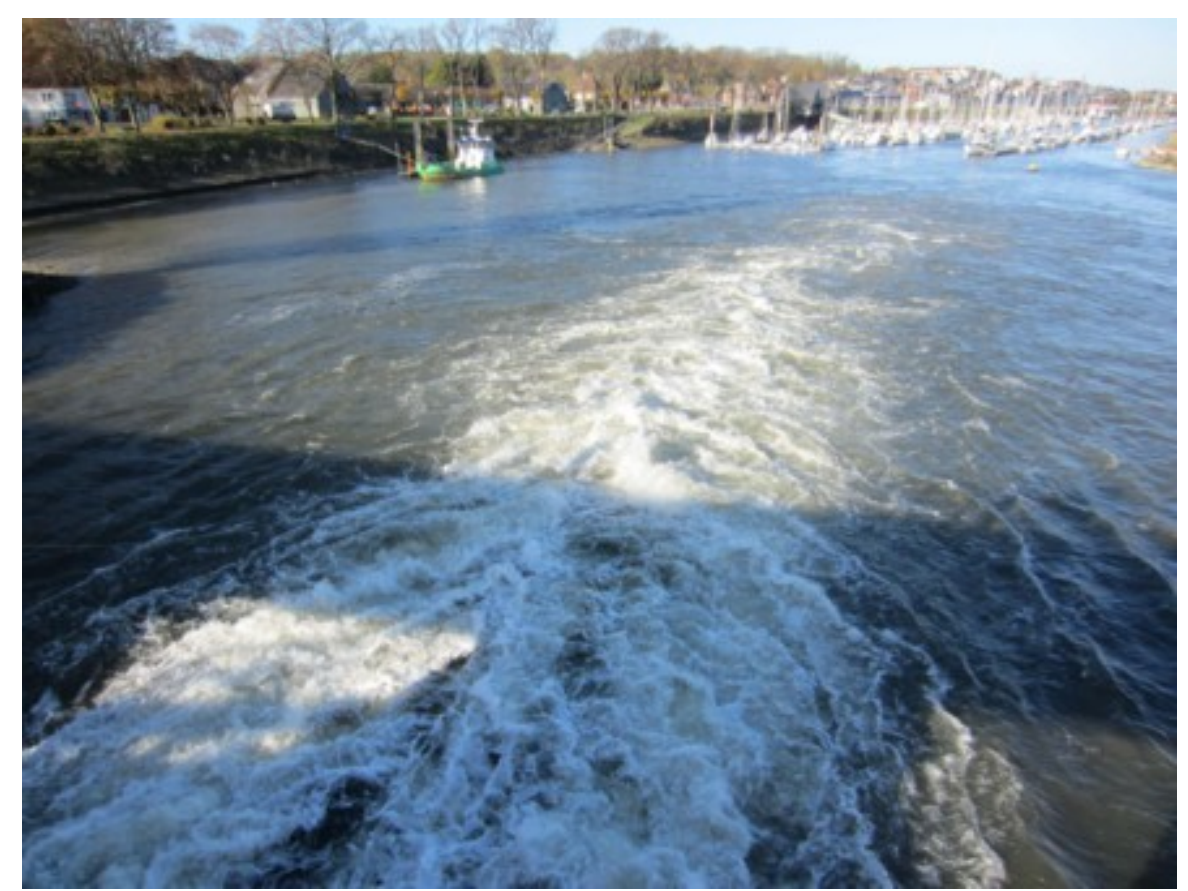

Figure 5. Zone de turbulence à l'aval du barrage inférieur.

iv) la phase 3 "chasses automatisées" organise des chasses pilotées par l'automate mais sous surveillance humaine; son objectif est de valider le comportement de l'automate selon les différentes configurations de marées, débits de la Somme, gestion des vannes et remplissage par entrée d'eau de la baie ; cette phase permet aussi un dernier affinage du protocole de gestion future des ouvrages; troisis cycles s'y succèdent : les 2 premiers prévoient une minimisation des risques avec un remplissage partiel par l'eau de la baie en vue de limiter des fermetures trop longues des vannes et sous régime de marées moyennes pour éviter des entrées d'eau trop importantes qui conduisent à des niveaux trop élevés dans le canal ; ces trois cycles prévus au cours du $1^{\text {er }}$ semestre 2014 visent respectivement à retrouver des fonds proches de ceux observés en avril 2013, à valider le maintien des fonds par les chasses et à valider le fonctionnement de l'automate dans des conditions particulières (marées de vives-eaux, mortes-eaux).

A l'issue de cette dernière phase, le protocole définitif de réalisation des chasses sera établi. Une nouvelle organisation du service exploitation sera mise en place ainsi qu'un suivi environnemental à long terme. La mise en place définitive des chasses dans la 


\section{XIII ${ }^{\text {èmes }}$ Journées Nationales Génie Côtier - Génie Civil \\ Dunkerque, 2-4 juillet 2014}

gestion courante sera conditionnée par l'obtention de l'arrêté préfectoral autorisant ces chasses.

\section{Discussion liée la mise en œuvre opérationnelle}

Avant toute action de chasse hydraulique, les conditions météorologiques, débitmétriques et les niveaux d'eau à l'amont et sur certains exutoires d'Abbeville sont vérifiés. En cas d'événements climatiques défavorables, l'action de chasse est alors ajournée.

Un comité de pilotage a été créé dès 2009. Il intégrait les partenaires institutionnels comme les services de l'Etat (Office National des Eaux et des Milieux Aquatiques, Direction Régionale de l'Environnement de l'Aménagement et du Logement, Direction Départementale des Territoires et de la Mer) et les élus concernés. Puis, dès 2010, la nécessité d'élargir ce comité de pilotage aux acteurs de terrain s'est fait sentir et un comité de suivi a été créé. Ce dernier intègre la Fédération de Pêche, l'association des "Pêcheurs à la ligne du Ponthieu", l'association de Vigilance sur les Inondations d'Abbeville et le Sport Nautique Valéricain. Ces acteurs de terrain ont participé aux différents suivis notamment celui des niveaux d'eau lors des différentes phases.

Une communication permanente est prévue lors de la mise en œuvre opérationnelle avec par exemple un bilan (débriefing) régulier après chaque essai de la première phase, de nombreux échanges avec la presse (communiqués, journaux, télévisions, ...), la distribution de tracts et la mise en place de panneaux d'information sur la zone d'étude.

\section{Références bibliographiques}

IN VIVO, ROYAL HASKONING, FISHPASS (2013). Suivi environnemental des chasses à Saint-Valery-sur-Somme - Etat initial (septembre 2012), Phase 1 : Tests de stockage et de mise en vitesse (avril 2013), Phase 2 : Séquences courtes. Rapport d'étude, octobre 2013.

SOGREAH Ingénierie, EDF (1994-1997). Projet baie de Somme - Eude sédimentologique sur modèle réduit. Rapport d'étude.

SOGREAH Ingénierie (2007). Projet baie de Somme - Aménagement du site de Saint-Valery-sur-Somme (3 ${ }^{\text {ème }}$ tranche) - Etude hydraulique (février 2007), Elaboration du protocole de suivi. Rapport d’étude, mars 2009. 
Thème 2 - Dynamique sédimentaire 